\title{
Increased energy intake entirely accounts for increase in body weight in women but not in men in the UK between 1986 and 2000
}

\author{
Peter Scarborough ${ }^{1 *}$, Melanie R. Burg ${ }^{2}$, Charlie Foster ${ }^{1}$, Boyd Swinburn ${ }^{3}$, Gary Sacks ${ }^{3}$, Mike Rayner ${ }^{1}$, \\ Premila Webster ${ }^{1}$ and Steven Allender ${ }^{1,3}$ \\ ${ }^{1}$ British Heart Foundation Health Promotion Research Group, Department of Public Health, University of Oxford, Old Road \\ Campus, Headington, Oxford OX3 $7 L F$, UK \\ ${ }^{2} J o h n$ Radcliffe Hospital, Headington, Oxford OX3 7LF, UK \\ ${ }^{3}$ Faculty of Health, Medicine, Nursing and Behavioural Sciences, Deakin University, Melbourne, VIC, Australia
}

(Received 17 May 2010 - Revised 3 November 2010 - Accepted 8 November 2010 - First published online 28 December 2010)

\section{Abstract}

There is debate over the casual factors for the rise in body weight in the UK. The present study investigates whether increases between 1986 and 2000 for men and women were a result of increases in mean total energy intake, decreases in mean physical activity levels or both. Estimates of mean total energy intake in 1986 and 2000 were derived from food availability data adjusted for wastage. Estimates of mean body weight for adults aged 19-64 years were derived from nationally representative dietary surveys conducted in 1986-7 and 2000-1. Predicted body weight in 1986 and 2000 was calculated using an equation relating body weight to total energy intake and sex. Differences in predicted mean body weight and actual mean body weight between the two time points were compared. Monte Carlo simulation methods were used to assess the stability of the estimates. The predicted increase in mean body weight due to changes in total energy intake between 1986 and 2000 was $4 \cdot 7$ (95\% credible interval $4 \cdot 2,5 \cdot 3) \mathrm{kg}$ for men and $6 \cdot 4(95 \%$ credible interval 5.9, 7.1) kg for women. Actual mean body weight increased by $7.7 \mathrm{~kg}$ for men and $5.4 \mathrm{~kg}$ for women between the two time points. We conclude that increases in mean total energy intake are sufficient to explain the increase in mean body weight for women between 1986 and 2000 , but for men, the increase in mean body weight is likely to be due to a combination of increased total energy intake and reduced physical activity levels.

\section{Key words: Body weight: Obesity: Diet: Physical activity: Energy intake}

The obesity epidemic in the UK is well documented - the prevalence of obesity in adults doubled between 1980 and $1991^{(1)}$ and has risen by more than $50 \%$ since, with more than half of all adults now either overweight or obese ${ }^{(2,3)}$. It is predicted that if current trends continue, then nearly $60 \%$ of the population could be obese by $2050^{(4)}$. Weight gain is a result of energy imbalance - total energy intake greater than total energy expenditure, where total energy expenditure consists of energy that is expended both by activity and by BMR. Both BMR and the amount of energy expended by activity are associated with body weight - the greater the mass of the body, the greater the energy required to move it around and the greater the BMR required to sustain it and vice versa. Often this relationship between body weight and energy expenditure is ignored, leading to false conclusions such as 'an increase in dietary energy intake of $100 \mathrm{~kJ} / \mathrm{d}$ will result in an increase in body weight of $x \mathrm{~kg}$ every year', when in reality the increase in energy intake will produce an increase in body weight in the short term, but the resultant increase in BMR and energy expended in activity will soon result in a new 'settling point' where body weight has reached a new, larger constant. A recent meta-analysis of studies that used the doubly labelled water technique has been used to estimate the relationship between body weight and total energy intake, taking into account the association between BMR, the amount of energy expended through activity and body weight ${ }^{(5)}$. The results of this analysis were applied to differences in mean energy intake in the US population in the early 1970s and early 2000s, and the researchers concluded that virtually all of the increase in mean body weight in the USA between these time points can be accounted for by changes in total energy intake ${ }^{(6)}$.

The relative contribution of increases in energy intake and decreases in physical activity levels to the increase in body

Abbreviation: USDA, US Department of Agriculture.

*Corresponding author: Dr P. Scarborough, email peter.scarborough@dphpc.ox.ac.uk 
weight in the UK is a subject of debate ${ }^{(1,7,8)}$. Untangling these influences is important, to help direct resources towards appropriate strategies to reverse the epidemic ${ }^{(9)}$. The aim of the present study is to assess the contributions of increases in mean total energy intake and decreases in mean physical activity levels to the increase in mean body weight in the UK between the mid-1980s and early 2000s, using similar methods to those applied to assess the change in mean body weight in the US population between the early 1970 s and early $2000 \mathrm{~s}^{(6)}$.

\section{Methods}

Sex-specific estimates of mean body weight, height and age were obtained from the Dietary and Nutritional Survey of British Adults, 1986- $7^{(10)}$, and the National Diet and Nutrition Survey, 2000-1 $1^{(11)}$ - two comparable surveys on a representative sample of a subpopulation of the UK (adults aged 19-64 years resident in Great Britain). Outliers in the height and weight variables were removed from both datasets (weight $\geq 150 \mathrm{~kg}$ and height $\geq 200 \mathrm{~cm}$ ). Individuals were also excluded if the measurement of body weight was unlikely to represent usual body weight - this included individuals who reported being ill during the survey period, and women who were either pregnant or breast-feeding. Respondents aged 16-18 years were excluded from the 1986-7 survey in order for the age structure of the two surveys to be comparable. After exclusion, the samples consisted of 1524 participants in the 1986-7 survey and 1315 participants in the 2000-1 survey.

Measures of total energy intake at the two time points were taken from food availability data, collected from food balance sheets prepared by the $\mathrm{FAO}^{(12)}$. These provide an estimate of the total availability of energy for the UK population ( $\mathrm{kJ} /$ person per $\mathrm{d}$ ) by assessing the amount of food commodities annually produced, imported and exported in the UK. The food availability data were adjusted for loss from spoilage and waste using methods developed by the US Department of Agriculture (USDA) ${ }^{(13)}$. Estimates of total energy intake for men and women separately were derived by applying the ratio of energy intake in men compared with women derived from the 1986-7 Dietary and Nutrition Survey to the adjusted food availability data (the ratio in 1986-7 (1.43) was virtually identical to the similar ratio recorded in 2000-1 (1.40)).

\section{Estimation of the influence of change in total energy intake}

The derivation of the linear equation linking body weight and total energy intake has been described and discussed elsewhere ${ }^{(5,14,15)}$. Briefly, the equations are derived from linear regressions on 1399 individuals from eight studies that collected data on body weight and total energy intake, collected using doubly labelled water techniques. A linear relationship between energy intake and body weight is assumed for individuals with constant physical activity levels. Each point on the regression line refers to a new 'settling point' of body weight that is achieved if energy intake is altered, and energy expenditure by BMR and physical activity increases/ decreases accordingly in response to the increase/decrease in body weight.

The equations for male and female are as follows:

$$
\begin{aligned}
& \text { Men : total energy intake }(\mathrm{kJ} / \mathrm{d}) \\
& \quad=93.0 \times \text { body weight }(\mathrm{kg})+4723 .
\end{aligned}
$$

Women : total energy intake $(\mathrm{kJ} / \mathrm{d})$

$$
=72.3 \times \text { body weight }(\mathrm{kg})+4873 \text {. }
$$

It is assumed that any variance around the regression line is due to measurement error or unmeasured variables (primarily physical activity levels).

These equations were applied to populations by substituting 'total energy intake' with 'mean total energy intake' and 'body weight' with 'mean body weight'. Using these equations, the predicted mean body weight of men and women in 1986 and 2000 was calculated from waste-adjusted food availability data. The difference in predicted mean body weights was compared with the difference in actual mean body weights taken from the nutrition survey samples. It was interpreted that the difference in predicted mean body weights represented the difference that can be accounted for by the changes in mean total energy intake, and that any remaining difference in actual mean body weights represented the difference that can be accounted for by the change in mean physical activity levels.

A sensitivity analysis was performed to assess the sensitivity of the results to the regression coefficients calculated using the meta-analysis of doubly labelled water studies. Here, a Monte Carlo simulation with 5000 iterations was performed, which allowed the regression parameters from the meta-analysis to vary following a normal distribution.

No human or animal subjects were used for the present study, which was therefore not submitted for ethical approval.

\section{Results}

Table 1 provides summary statistics for the two nutrition survey samples, and shows that between 1986 and 2000, mean body weight in men increased by $7.7 \mathrm{~kg}$, and in women, it increased by $5.4 \mathrm{~kg}$. The two samples were not entirely comparable - for example, the latter sample was slightly older and contained substantially fewer women from the manual social classes.

After adjusting the UK food availability data for loss, total energy available/person per $\mathrm{d}$ for men was $10626 \mathrm{~kJ}$ $(2530 \mathrm{kcal})$ in 1986 , and $11063 \mathrm{~kJ}(2634 \mathrm{kcal})$ in 2000 . For women, this was $7446 \mathrm{~kJ}(1772 \mathrm{kcal})$ in 1986 and $7911 \mathrm{~kJ}$ (1883 kcal) in 2000. Table 2 also shows equivalent estimates using unadjusted food availability data, estimates from $7 \mathrm{~d}$ weighed food dairies collected for the nutrition surveys and estimates from food purchase surveys.

Using the equations described earlier, the difference in predicted mean body weights between 1986 and 2000 
Table 1. Summary statistics comparing the samples of the $1986-7$ and $2000-1$ dietary surveys

\begin{tabular}{lccr}
\hline & $1986-7$ & $2000-1$ & \multicolumn{1}{c}{$P$} \\
\hline Men & & & \\
$n$ & 822 & 629 & \\
Mean age (years) & 40 & 42 & 0.0027 \\
Non-white (\%) & $4 \cdot 2$ & $5 \cdot 2$ & 0.6421 \\
Manual social class (\%) & 44.7 & $45 \cdot 6$ & 0.7414 \\
Mean height (cm) & 174 & 176 & $<0.0001$ \\
$\quad$ Mean weight (kg) & $76 \cdot 2$ & 83.9 & $<0.0001$ \\
Women & & & \\
$n$ & 702 & 686 & \\
Mean age (years) & 41 & 43 & 0.0029 \\
Non-white (\%) & 3.8 & $6 \cdot 0$ & 0.0643 \\
Manual social class (\%) & $44 \cdot 7$ & 32.5 & $<0.0001$ \\
Mean height (cm) & 162 & 162 & 1.0000 \\
Mean weight (kg) & 63.5 & 68.9 & $<0.0001$ \\
\hline
\end{tabular}

(i.e. the difference that is due to changes in mean total energy intake) was $4.7 \mathrm{~kg}$ for men. Actual population weight gain over this time period (estimated using the dietary surveys) was $7.7 \mathrm{~kg}$, suggesting that change in mean body weight was due to both increased mean total energy intake and decreased mean physical activity levels. For women, the difference in predicted mean body weights between 1986 and 2000 was $6.4 \mathrm{~kg}$. Actual population weight gain over this time period was only $5.4 \mathrm{~kg}$, suggesting that an increase in total energy intake was sufficient to explain the increase in body weight over this time period.

Table 3 shows the results of the sensitivity analysis, and indicates that the results are fairly robust to changes in the regression parameters calculated for the aforementioned equations. For both men and women, the results across the entire of the $95 \%$ credible intervals do not alter the conclusion that increase in body weight in men is due to both increases in total energy intake and decreases in physical activity levels, but for women, the increase in body weight can be explained by increases in total energy intake alone.

Table 2. Estimates of mean total energy intake $(\mathrm{kJ} /$ person per d) from unadjusted food balance sheets, adjusted food balance sheets and dietary surveys

\begin{tabular}{lrr}
\hline & 1986 & 2000 \\
\hline Men & & \\
Unadjusted food balance sheets* & 16006 & 16695 \\
Adjusted food balance sheets $†$ & 10626 & 11063 \\
Dietary surveys $\ddagger$ & 10410 & 9900 \\
Food purchase estimates§ & 10232 & 8651 \\
Women & & \\
Unadjusted food balance sheets & 11218 & 11840 \\
Adjusted food balance sheets $†$ & 7446 & 7911 \\
Dietary surveys & 7295 & 7080 \\
Food purchase estimates§ & 7156 & 6049 \\
\hline
\end{tabular}

* Unadjusted food balance sheets present the estimate of available energy from food

†Adjusted food balance sheets present the estimate of energy availability adjusted for wastage.

$\ddagger$ Dietary surveys are measures of self-reported food intake using $7 \mathrm{~d}$ weighed food diaries.

$\S$ Food purchase estimates are measures derived from purchases of foods consumed within the home adjusted for men and women using a ratio of 1.43 between male and female consumption.
Table 3. Sensitivity of the results to regression parameters

\begin{tabular}{lcc}
\hline & $\begin{array}{c}95 \% \text { Credible interval } \\
\text { in predicted change in } \\
\text { mean body wt due to } \\
\text { total energy intake }(\mathrm{kg})\end{array}$ \\
\hline Men & 7.7 & $4.2,5.3$ \\
Women & 5.4 & $5.9,7.1$ \\
\hline
\end{tabular}

\section{Discussion}

The results presented here suggest that the increase in body weight in the UK between the mid-1980s and early 2000s has different causal factors for men and women. For men only part of the increase in body weight can be ascribed to the increase in energy intake, suggesting that reductions in physical activity levels have also played a role. For women, increases in energy intake explain all of the increase in body weight, suggesting that this was the dominant causal factor for increases in body weight over this time period. In order to view these results in context, they should be compared with trend data in both total energy intake and physical activity levels. Both of these variables are difficult to accurately assess, and therefore trends in either are usually provided from updatable proxy measures. In the case of total energy intake, regular British trend data are available for two different proxy measures (food availability and food purchase data), and infrequent trend data are available from dietary surveys, which are designed to directly measure food consumption. Each of these data sources has its own limitations. Food availability data (as is used in the present study) overestimate total energy intake, as they do not account for food wastage at all points of the food chain. In this study, this limitation has been addressed by adjusting the data for loss and wastage at different stages of the food chain ${ }^{(13)}$, but this adjustment will inevitably result in measurement error. Food purchase data also do not take account of food wastage at the consumer level, and most food purchase studies only include foods purchased for consumption within the household. For example, the National Food Survey estimates of energy consumption in 1986 and 2000 in the UK are presented in Table 2, which suggest that energy consumption fell between these two time points ${ }^{(16)}$. But only foods purchased for consumption within the household are included, and using the results as estimates of total energy intake is therefore subject to differential bias if consumption of foods outside of the house increased between 1986 and 2000. Data collected using dietary surveys are capable of providing a direct estimate of total energy intake, but they are subject to differential underreporting (individuals with an unhealthy diet are more likely to under-report than individuals with a healthy diet, and all individuals are more likely to under-report consumption of unhealthy foods than healthy foods $)^{(17-19)}$. The differing limitations of the measures of total energy intake are important to take into account, as different measures of total energy intake produce very different results. It has been shown that trends in total energy intake in the UK derived from food availability data and food survey data are opposed to each other, with 
energy intake trends increasing when food availability data are used and decreasing when food survey data are used ${ }^{(20,21)}$. As shown in Table 2 , the estimates of mean total energy intake provided by the dietary surveys at the two time points suggest that energy intake decreased between the mid-1980s and early 2000s. Using the dietary survey data in the calculations reported in the present study would produce a very different interpretation - that change in mean body weight over the time period for both sexes was due entirely to decreases in physical activity, and the corresponding decrease in mean total energy intake over the same period has not been enough to offset the increase in mean body weight. Indeed, a similar conclusion has previously been made from an investigation of dietary survey data ${ }^{(1)}$. In the present study, we have used food availability data rather than dietary survey data this is because we believe that the biases introduced by the limitations of food availability data are likely to be more stable over time than those introduced by dietary survey data, meaning that trend data from food availability data are likely to provide a better reflection of true trends.

The conclusion that the increase in mean body weight is partially due to decreases in physical activity over the same time period is inconsistently supported by some trend data in population measures of physical activity. As with measures of nutrition intake, assessing population levels of physical activity behaviour requires accurate measurement of a range of physical activity-related domains. These discrete behaviours include sport, recreational and occupational activities, heavy domestic activity (gardening and housework) and active travel. They are usually assessed by self-report or more recently objective measures (e.g. accelerometery). Recent trends from the Health Survey for England in overall physical activity have suggested a gradual increase in the proportion of adults achieving current public health recommendations (assessed by self-report) $^{(22)}$. In 1997, 32\% of men met the recommendations, increasing to $42 \%$ in 2008 , and for women, $21 \%$ met the recommendations in 1997 , increasing to $31 \%$ in 2008. However, there has been a consistent decline in active travel (walking and cycling) since $1988^{(23)}$. Stamatakis et $a l^{(24)}$ challenged the misperception that physical activity levels are in decline; despite decreases in occupational physical activity, they reported that there was a clear upward trend in sports participation from 1999 to 2004. Overall, there are inconsistencies in the temporal trends of population physical activity survey data, which confound possible explanations for changes in body weight. One possible explanation for the contribution of decreased physical activity levels to weight gain may be the impact of increasing sedentary behaviours across the population. Although no longitudinal data exist for this area in the UK, one study has reported that men and women spent at least $7 \mathrm{~h} / \mathrm{d}$ being sedentary ${ }^{(25)}$, using estimates derived from heart rate. Clearly, sedentary behaviours for adults such as television watching or computer use are more available and in themselves are perhaps more persuasive behavioural choices, and perhaps may have an impact on opportunities and desire to be physically active.

The results presented in the present study are dependent upon the equations linking body weight and total energy intake in individuals, which were derived from meta-analyses of doubly labelled water studies. Alternative equations have been derived using theoretical associations between BMR, physical activity levels, weight and height, with additional parameterisation using data from eight longitudinal weightloss studies ${ }^{(26)}$. Models utilising these equations are freely accessible from the US Department of Health and Human Services website (http://www2.niddk.nih.gov/NIDDKLabs/ LBM/HallAJCN2008.htm). The alternative equations display a similar slope to those used in the analyses reported here, and if a low level of physical activity levels is assumed in the UK between the 1980s and 2000s, then the equations produce similar conclusions - that increases in total energy intake entirely explain the increase in body weight in women but not in men.

The method used here is subject to a number of limitations that should be addressed. The conclusions are dependent upon the assumption that the equations linking body weight and total energy intake in individuals are adaptable to populations, which could result in the individualistic fallacy (the opposite of the ecological fallacy, assuming that an association measured at the individual level is equivalent at the population level) ${ }^{(27)}$. Both the ecological and the individualistic fallacy are a result of non-adjustment for confounding variables that influence the relationship at one level but not at the other level. The equations linking body weight and energy intake are unlikely to be heavily affected by confounding variables that have not been adjusted for (at either level) since the equation operates at the physiological level, where the contributing factors to change in body weight are either already included in the model (total energy intake) or considered to account for some of the variance around the regression line (physical activity levels). As can be seen in Fig. 1, for both sexes, the predicted mean body weight was lower than the actual measured mean body weight, and this may be evidence of misspecification of the equations, or could be a result of underestimates of total energy intake for the adult population of the UK (since food availability data include food that is available for consumption by children and by elderly people, who are likely to consume less than adults aged 19-64 years). Since the outcome of interest was 'change in predicted mean body weight', the misspecification at the two time points should cancel out. A further limitation is the change in the social distribution of women between the two dietary surveys that provided estimates of actual body weight for the two time periods. Obesity in women in Great Britain is socially patterned, with higher levels in the lower social classes ${ }^{(2,28)}$. Therefore, the increase in mean body weight between the two time periods estimated by the differences from the dietary surveys is likely to be an underestimate of the true increase in mean body weight within women in Britain; consequently, the proportion of this increase that is due to increases in total energy intake may have been overestimated. Similarly, the average height of the male sample in 2000 was slightly larger than that in 1986, which may account for some of the difference in body weight between the two time points. 


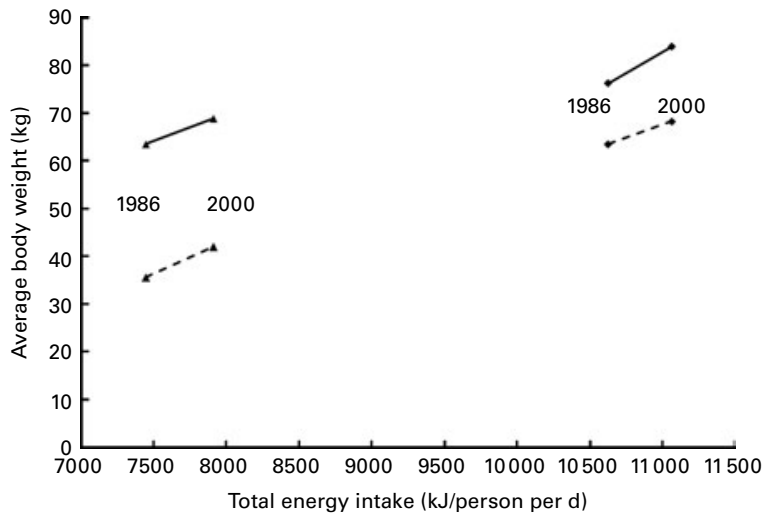

Fig. 1. Comparison of predicted change in body weight between 1986 and 2000 with actual change in body weight between 1986 and 2000. Body weight is predicted from loss-adjusted food availability data. $\rightarrow$, Actual change (men); - - , predicted change (men); $\longleftarrow$, actual change (women); -r, predicted change (women).

Another limitation is the inexact adjustment for food wastage used in the analyses. A method developed by the USDA was used here ${ }^{(13)}$, rather than a method developed by the Department for Environment, Food and Rural Affairs, which may be more relevant to the UK setting ${ }^{(29)}$. There were two reasons for this choice - first, using the USDA methods produces comparable results with an earlier analysis of changes in body weight in the USA ${ }^{(6)}$. Second, the USDA method is better designed for use with food availability data, which is used for the analyses presented here. The USDA method applies a conversion factor directly to each food category, which accounts for 'subsequent processing, trimming, shrinkage or loss between the farm and retail levels ${ }^{(13)}$. However, the Department for Environment, Food and Rural Affairs method provides a conversion for sixty-five food categories not used for these analyses that relate to foods as bought and account for wastage and spoilage within the house ${ }^{(29)}$. While neither method adjusts for wastage at all stages of the food chain, the USDA method is more directly applicable to the data used in the analyses reported in this study. Because the USDA method does not account for wastage at the retail level or at the consumer level, it is possible that changes in retail- or consumer-level food waste between 1986 and 2000 could have an impact on the results reported here. Using alternative equations linking body weight and energy intake, Hall et al. ${ }^{(30)}$ have also investigated the increase in body weight between the 1970s and the present day in the USA. This study produced similar results to those produced by Swinburn et al., but came to a different conclusion - that the amount of food that is wasted in the USA must be increasing. For the analyses that have been presented here, we have adjusted for food wastage within broad food groups, but we have assumed that within each broad food group, the proportion of food that is wasted in the UK has remained reasonably constant between 1986 and 2000 . If this is not the case, and wastage of food in some (or all) food groups has increased, then our analyses will have overestimated the proportion of the increase in body weight that can be explained by increases in energy intake.
The results presented here are comparable with previous work using US data on food availability and increases in body weight between the 1970s and early 2000s. Using similar body weight-energy intake equations and a similar analysis design, it has been shown that increases in total energy intake over this time period were sufficient to explain increases in body weight for both children and adults ${ }^{(6)}$. The results were not stratified by sex, as has been the case here, but the difference in the US results and in the male UK results suggests that the obesity epidemic that has affected most Western developed nations may not have universal causal factors, implying that public health strategies to tackle obesity in the USA may not be suited to tackle obesity in the UK. Similarly, the results presented here suggest that the causal factors for population weight gain for male and female adults in the UK may not be exactly equivalent. This has implications for proposed interventions designed to tackle adult obesity in the UK. Obviously, effective interventions to improve both physical activity levels and dietary quality would be beneficial to men and women in the UK, but any effective sustainable intervention must result in a future energy intake/energy expenditure balance that is acceptable to the UK population. The results presented here suggest that to achieve such a balance, initiatives addressing the obesogenicity of the food environment will be beneficial for both men and women in the UK, and interventions increasing physical activity levels in men are also required. This could result in a balance that was achieved in the recent past, and hence may be acceptable to a future UK population.

Further research in this area could address other populations that have recently experienced a change in mean body weight. This could include children in the UK or populations from other countries. The methods employed here are easily reproducible, requiring only estimates of mean body weight from comparable nationally representative samples at two time points and adaptation of food availability data provided by the FAO. Replication of the results for other populations could provide greater insight into the aetiology of the obesity epidemic in developed nations, and it would also provide greater scrutiny of the limitations involved with this methodology (e.g. by comparing conclusions with proxy trends in total energy intake and physical activity levels in different countries).

\section{Acknowledgements}

P. S., C. F., M. R. and S. A. are supported by the British Heart Foundation. The authors declare no conflicts of interest. All of the authors contributed to the drafting of the manuscript. The idea for the project and the analysis plans were created by P. S., B. S., G. S. and S. A. The analysis was conducted by M. R. B. and P. S.

\section{References}

1. Prentice A \& Jebb S (1995) Obesity in Britain: gluttony or sloth? BMJ 311, 437-439. 
2. Joint Health Surveys Unit (2008) Health Survey for England 2006. Cardiovascular Disease and Risk Factors in Adults Leeds: The Information Centre.

3. Allender S, Peto V, Scarborough P, et al. (2008) Coronary Heart Disease Statistics 2008. London: British Heart Foundation.

4. Butland B, Jebb S, Kopelman P, et al. (2007) Foresight. Tackling Obesities: Future Choices - Project Report. London: Government Office for Science.

5. Swinburn B, Sacks G, Lo S, et al. (2009) Estimating the changes in energy flux that characterize the rise in obesity prevalence. Am J Clin Nutr 89, 1723-1728.

6. Swinburn B, Sacks G \& Ravussin E (2009) Increased food energy supply is more than sufficient to explain the US epidemic of obesity. Am J Clin Nutr 90, 1453-1456.

7. Westerterp K (2008) Physical activity as determinant of daily energy expenditure. Physiol Behav 93, 1039-1043.

8. Kopelman P \& Grace C (2004) New thoughts on managing obesity. Gut 53, 1044-1053.

9. Swinburn B, Caterson I, Seidell J, et al. (2004) Diet, nutrition and the prevention of excess weight gain and obesity. Public Health Nutr 7, 123-146.

10. Gregory J, Foster K, Tyler H, et al. (1990) The Dietary and Nutritional Survey of British Adults. London: HMSO.

11. Henderson L, Gregory J \& Swan G (2002) The National Diet and Nutrition Survey: Adults Aged 19-64. London: HMSO.

12. FAOSTAT (2009) Food and Agriculture Organization Statistical Database. Geneva: FAO. http://faostat.fao.org/default. aspx (accessed December 2009)

13. Kantor L (1998) A Dietary Assessment of the U.S. Food Supply: Comparing Per Capita Food Consumption with Food Guide Pyramid Serving Recommendations. Washington, DC: U.S. Department of Agriculture.

14. Swinburn B, Sacks G \& Ravussin E (2010) Estimating the quantitative relationship between changes of food energy intake and body weight (response). Am J Clin Nutr 91, 817, (letter)

15. Hall K \& Chow C (2010) Estimating the quantitative relationship between changes of food energy intake and body weight. Am J Clin Nutr 91, 816, (letter).

16. Department for Environment, Food \& Rural Affairs (2001) National Food Survey 2000. London: The Stationery Office.
17. Lichtman S, Pisarska K, Berman E, et al. (1992) Discrepancy between self-reported and actual caloric intake and exercise in obese subjects. $N$ Engl J Med 327, 1893-1898.

18. Becker W \& Welten D (2001) Under-reporting in dietary surveys - implications for development of food-based dietary guidelines. Public Health Nutr 4, 683-687.

19. Scagliusi F, Ferriolli E, Pfrimer K, et al. (2008) Under-reporting of energy intake is more prevalent in a healthy dietary pattern cluster. Br J Nutr 100, 1060-1068.

20. Stephen A (2001) Energy intake: what we know and what we don't. In A National Dialogue on Healthy Body Weights. Summary of Proceedings. Toronto: Canadian Institute of Health Research.

21. Stephen A, Bramwell G \& Reid J (2005) Trends in energy and fat intake in the US and UK over 8 decades. Poster Presentation at the 18th International Congress of Nutrion D, South Africa.

22. Joint Health Surveys Unit (2009) Health Survey for England 2008. Physical Activity and Fitness. Leeds: The Information Centre.

23. Department of Transport (2007) Transport Statistics Bulletin. National Travel Survey 2006. London: Department of Transport.

24. Stamatakis E, Ekelund U \& Wareham N (2007) Temporal trends in physical activity in England: the Health Survey for England 1991 to 2004. Prev Med 45, 416-423.

25. Ekelund U, Brage S, Besson $\mathrm{H}$, et al. (2008) Timespent being sedentary and weight gain in healthy adults: reverse orbidirectional causality? Am J Clin Nutr 88, 612-617.

26. Hall K \& Jordan P (2008) Modeling weight-loss maintenance to help prevent body weight regain. Am J Clin Nutr 88, $1495-1503$.

27. Subramanian S, Jones K, Kaddour A, et al. (2009) Revisiting Robinson: the perils of individualistic and ecologic fallacy. Int J Epidemiol 38, 342-360.

28. Scottish Centre for Social Research (2009) The Scottish Health Survey 2008. Edinburgh: The Scottish Government.

29. Office for National Statistics (2008) Family Food in 2007. London: The Stationery Office.

30. Hall K, Gup J, Dore M, et al. (2009) The progressive increase of food waste in America and its environmental impact. PLoS One 4, e7940. 